\title{
In vivo bradykinin $B 2$ receptor activation reduces renal fibrosis
}

\author{
Joost P. Schanstra, ${ }^{1}$ Eric Neau, ${ }^{1}$ Pascale Drogoz, ${ }^{1}$ Miguel A. Arevalo Gomez, ${ }^{2}$ \\ José Miguel Lopez Novoa, ${ }^{2}$ Denis Calise, ${ }^{1}$ Christiane Pecher, ${ }^{1}$ Michael Bader, ${ }^{3}$ \\ Jean-Pierre Girolami, ${ }^{1}$ and Jean-Loup Bascands ${ }^{1}$
}

${ }^{1}$ Inserm U388, Institut Louis Bugnard, Toulouse, France

${ }^{2}$ School of Medicine, University of Salamanca, Salamanca, Spain

${ }^{3}$ Max Delbrück Center, Berlin-Buch, Germany

\begin{abstract}
Angiotensin-converting enzyme (ACE) inhibitors reduce the progression of various fibrotic renal diseases both in humans and in animal models. Unilateral ureteral obstruction (UUO) is an animal model of accelerated renal tubulointerstitial fibrosis that is attenuated by ACE inhibition. Although ACE inhibitors increase bradykinin concentrations in addition to their effect on angiotensin II formation, the role of bradykinin in renal fibrosis has not been studied. We show here that genetic ablation $\left(B 2^{-/-}\right.$mice) or pharmacological blockade of the bradykinin $\mathrm{B} 2$ receptor increases UUO-induced interstitial fibrosis in mice, whereas transgenic rats expressing increased endogenous bradykinin show reduced UUO-induced interstitial fibrosis. The increased interstitial fibrosis in $\mathrm{B2}^{-/-}$mice was accompanied by a decreased activity of plasminogen activators (PAs) and metalloproteinase-2 (MMP-2), enzymes involved in ECM degradation, suggesting that the protective effects of bradykinin involve activation of a $\mathrm{B} 2$ receptor/PA/MMP-2 cascade. This ability of bradykinin to increase PA activity was confirmed in primary culture proximal tubular cells. Thus, in both mice and rats, bradykinin $\mathrm{B} 2$ receptor activation reduces renal tubulointerstitial fibrosis in vivo, most likely by increasing ECM degradation.
\end{abstract}

J. Clin. Invest. 110:371-379 (2002). doi:10.1172/JCI200215493.

\section{Introduction}

Most forms of chronic renal disease tend to progress to end-stage glomerulosclerosis and tubulointerstitial fibrosis (1). Tubulointerstitial fibrosis is preceded by marked infiltration of the tubulointerstitium by inflammatory mediators, interstitial fibroblast proliferation, and tubular dilation, resulting finally in increased ECM protein deposition in the tubular interstitium (2). In most renal diseases the degree of tubulointerstitial changes observed in the early stage of different nephropathies predicts the long-term outcome of renal function (3).

Unilateral ureteral obstruction (UUO) in mice or rats is a well-established nonimmune inflammatory experimental model resulting in tubulointerstitial fibrosis in the obstructed kidney. In humans, chronic but also

Received for publication March 21, 2002, and accepted in revised form June 11, 2002.

Address correspondence to: Jean-Loup Bascands, Inserm U388, Institut Louis Bugnard, CHU Rangueil, 31052 Toulouse, France. Phone: 33-05-61-32-22-11; Fax: 33-05-62-17-25-54; E-mail: bascalou@toulouse.inserm.fr.

Conflict of interest: No conflict of interest has been declared.

Nonstandard abbreviations used: unilateral ureteral obstruction (UUO); angiotensin-converting enzyme (ACE); angiotensin type I (AT1); plasminogen activator (PA); urokinase-type PA (uPA); tissue-type PA (tPA); periodic acid-Schiff (PAS); proliferating cell nuclear antigen (PCNA); $\alpha$-smooth muscle actin ( $\alpha$-SMC); Krebs Henseleit buffer (KHB); monocyte chemoattractant peptide-1 (MCP-1); PA inhibitor type 1 (PAI-1); $N$-methyl-D-aspartate (NMDA). acute ureteral obstruction occurs in different kidney diseases at all ages. Most of the time it is congenital in newborns or induced by trauma or prostatic hypertrophy in adults. Without an appropriate treatment, these obstructive uropathies may progress to end-stage renal disease. In acute ureteral obstruction, the relief of the obstruction is obviously the most efficient therapy (4). However, this is not always feasible, and when detected too late, even relief of the obstruction does not stop the progression of tubulointerstitial fibrosis. Indeed, in a model of temporary UUO in the neonatal rat, despite the relief of the obstruction, growth of the postobstructed kidney was impaired and interstitial fibrosis was observed leading to renal insufficiency in adulthood (5).

In animal models of UUO it has been shown that angiotensin-converting enzyme (ACE) inhibitors and, to a lesser extent, angiotensin type I (AT1) receptor antagonists, prevent the progression of tubulointerstitial fibrosis $(6,7)$. ACE converts angiotensin I into angiotensin II and degrades bradykinin (8). Moreover, it has been found that ACE has a higher affinity for bradykinin than for angiotensin I (9) and that ACE inhibition significantly increases bradykinin concentrations (10).

A number of experimental and clinical studies have shown the therapeutic relevance of ACE inhibitors in slowing the progression of various renal diseases (11-13). Although in these studies it has been strongly suggested that bradykinin could participate actively in the beneficial effects of ACE inhibitors, no functional studies of the role of bradykinin and its $G$ 
protein-coupled B2 receptor in renal tubulointerstitial fibrosis have been reported. That is the reason why we studied the functional role of the bradykinin B2 receptor in the development of tubulointerstitial fibrosis caused by UUO.

We report here that renal tubulointerstitial fibrosis induced by UUO is significantly higher in $\mathrm{B} 2$ receptor knockout $\left(B 2^{-{ }^{-}}\right)$mice than in wild-type $\left(B 2^{+/+}\right)$mice. Confirming this observation, UUO-induced tubulointerstitial fibrosis was reduced in transgenic rats overexpressing the tissue kallikrein gene (expressing increased endogenous bradykinin). It was found that $B 2^{-/-}$mice have a lower plasminogen activator (PA) (comprising urokinase-type PA [uPA] and tissue-type PA [tPA]) activity associated with a decrease in metalloproteinase (MMP-2) activity, which can explain the increased UUO-induced tubulointerstitial fibrosis in $B 2^{-/-}$mice. The ability of bradykinin to increase PA activity was confirmed in primary culture renal proximal tubular cells. The present study demonstrates, we believe for the first time in vivo, that bradykinin B2 receptor activation plays a protective role in renal tubulointerstitial fibrosis.

\section{Methods}

Animals. B2 $2^{-/-}$mice were generously provided by F. Hess and T. MacNeil (Merck \& Co. Inc., Rahway, New Jersey, USA) (14). $B 2^{-/-}$mice were originally on a mixed genetic background (J129sv $\times \mathrm{C} 57 \mathrm{Bl} / 6 \mathrm{~J})$. We have backcrossed (ten times) the $\mathrm{B2} 2^{-/-}$mice to $\mathrm{C} 57 \mathrm{Bl} / 6 \mathrm{~J}$ and have therefore used $\mathrm{C} 57 \mathrm{Bl} / 6 \mathrm{~J}$ as control mice. Microsatellite analyses confirmed the $\mathrm{C} 57 \mathrm{Bl} / 6 \mathrm{~J}$ genetic background of the mice by backcrossing generated $\mathrm{B2}^{-\gamma^{-}}$mice (Nucleis, Angers, France). Furthermore, the mice are housed in a pathogen-free environment. Transgenic rats overexpressing the human tissue kallikrein gene, TGR(hKLK1), and their transgene negative littermates were generated as described previously (15).

Experimental protocols. Male mice or rats at the age of 8 weeks were used in these experiments, ten mice or five rats in each group. The unilateral ureteral ligation was performed as follows: under oxygen-isoflurane anesthesia, through a longitudinal, left abdominal incision, the ureter was exposed and ligated with a $6 / 0$ nylon (4/0 for rats) thread at the uretero-pelvic junction. In sham operations, the ureter was exposed but not ligated and repositioned. Mice and rats were maintained on a standard mouse or rat chow and tap water. All treatments were initiated 1 day before obstruction and continued throughout the time of obstruction (3 hours to 14 days). B2 receptor antagonist HOE-140 $(500 \mu \mathrm{g} / \mathrm{kg}$; HOECHST) was injected daily by one subcutaneous injection. At the end of the different protocols, mice or rats were sacrificed, and the kidneys were removed and divided in different parts according to the different protocols employed. All experiments reported were conducted in accordance with the NIH guide for the care and use of laboratory animals and were approved by a local animal care and use committee.
Immunohistochemistry. Kidneys were fixed in Carnoy's solution, dehydrated, embedded in paraffin, and then sectioned at $3 \mu \mathrm{m}$ and subjected to the following analyses. Once deparaffinized and rehydrated, the kidney sections were stained with Sirius red and periodic acidSchiff (PAS) to evaluate interstitial collagen deposition and tubular atrophy, respectively. Immunohistochemical studies for the detection of fibronectin, collagen type I, III, IV, macrophages, the proliferating cell nuclear antigen (PCNA), and $\alpha$-smooth muscle actin $(\alpha-S M C)$ for the detection of myofibroblasts were performed as follows. Deparaffinized sections were incubated with $3 \%$ hydrogen peroxide to block the endogenous peroxidase activity. The sections were then incubated for 1 hour at room temperature in a humidified atmosphere with the following primary Ab's: goat polyclonal anti-human fibronectin (SC-6953; Santa Cruz Biotechnology Inc., Santa Cruz, California, USA), rabbit polyclonal anti-rat collagen type I (AB755; Chemicon International, Temecula, California, USA), rabbit anti-human collagen III and IV (T59105R and T59106R, respectively; Interchim, Montluçon, France), the rat mAb to mouse F4/80 (RM2900; Caltag Laboratories Inc., Burlingame, California, USA) for macrophage detection, and the monoclonal mouse anti-human $\alpha$-SMC (DAKO EPOS method, U7033; DAKO S.A., Trappes, France). For fibronectin and collagen I, the sections were sequentially processed with the goat (fibronectin) or rabbit (collagen I) ABC Staining System (Santa Cruz Biotechnology Inc.), which uses $3,3^{\prime}$-diaminobenzidine as chromogen. For the visualization of collagen III and IV and F4/80, the DAKO Envision System was used (DAKO S.A.). Cell proliferation was assessed by detection of PCNA using the Zymed PCNA staining kit (Zymed Laboratories Inc., San Francisco, California, USA). Sections were counterstained with hematoxylin. For all samples, negative controls for the immunohistochemical procedures included substitution of the primary Ab with nonimmune sera. The presence of myofibroblasts was analyzed on PCNA adjacent sections to be able to compare both localizations. Glomeruli and vasculature were excluded from the evaluation in the present study.

Histomorphometric analysis. Analyses were performed by an operator unaware of the origin of each kidney section. Under a light microscope (Nikon Eclipse 600) at $\times 200$ magnification, 20 nonoverlapping fields (to obtain around $90 \%$ of the kidney section) per kidney section were captured with a numeric camera (Cool SNAP; RS Photometrics, Tucson, Arizona, USA) connected to the microscope. Quantitative analysis of the pictures was performed by using the Adobe PhotoShop 5.5 software, which allows counting of the pixels stained specifically (red for Sirius red and brown for the immunohistochemical studies). The results are given in percentage of specific colored pixels per total number of pixels of the 20 fields, representing thus the surface occupied by the analyzed marker. For PCNA and $\alpha$-SMC detection, marked cells were counted. 
RNA preparation and Northern blot analysis. Total RNA from snap-frozen kidney sections was extracted using the RNeasy Midi kit (QIAGEN Inc., Valencia, California, USA). Glyoxal and dimethyl sulfoxide denatured RNA $(20 \mu \mathrm{g})$ was separated on a phosphate-buffered $1 \%$ agarose gel and transferred under vacuum with a blotting device on a positively charged polyamide membrane (Schleicher \& Schuell Inc., Keene, New Hampshire, USA). The cDNA probes (between 200 and $350 \mathrm{bp}$ ) were obtained by RT-PCR, verified by sequencing, and radiolabeled with $\left[\alpha-{ }^{32} \mathrm{P}\right] \mathrm{dATP}$ using the Strip-EZ DNA Kit (Ambion Inc., Austin, Texas, USA). Hybridization was performed in Ultrahyb buffer (Ambion Inc.). Densitometric analysis was performed as described (16).

Primary culture of mouse renal proximal tubular cells. Mouse proximal tubular cells were obtained by an extension of a method described previously (17). Briefly, after removal of the kidneys, the kidney cortex was minced in HBSS at $4^{\circ} \mathrm{C}$ and incubated two times for 20 minutes at $37^{\circ} \mathrm{C}$ with $0.2 \mathrm{mg} / \mathrm{ml}$ collagenase solution supplemented by $1 \mathrm{mg} / \mathrm{ml}$ BSA. After centrifugation ( 5 minutes at $135 \mathrm{~g}$ ), the pellet was washed two times with HBSS and two times with HBSS containing 1\% BSA. To obtain homogenous populations of nephron segments, the mixture of tubules was resuspended in $42 \%$ Percoll made isotonic with concentrated $(10 \times)$ Krebs Henseleit buffer (KHB), pH 7.3 - 1.18 M NaCl, 47 mM KCl, 100 mM HEPES, $200 \mathrm{mM}$ cyclamic acid, $1.26 \mathrm{mM} \mathrm{MgSO}_{4}, 11.4$ $\mathrm{mM} \mathrm{KH}_{2} \mathrm{PO}_{4}$, and $50 \mathrm{mM}$ glucose - and centrifuged $\left(34,900 \mathrm{~g}\right.$ for 30 minutes at $\left.4^{\circ} \mathrm{C}\right)$. The F4 layer, containing the proximal tubules, was suspended in culture medium (DMEM/HAM F12) supplemented with $5 \% \mathrm{FCS}, 10 \mu \mathrm{g} / \mathrm{ml}$ insulin, $5 \mathrm{mM}$ transferrin, $10 \mathrm{ng} / \mathrm{ml}$ EGF, $0.1 \mu \mathrm{M}$ dexamethasone, and $5 \mu \mathrm{g} / \mathrm{ml}$ triodothyronin, and plated at a concentration of $1.6 \mathrm{mg}$ of protein per $100 \mathrm{~mm}^{2}$ of a Petri dish coated with collagen. The proximal tubular origin and epithelial cell type was confirmed by positive alkaline phosphatase activity and cytokeratin staining (data not shown). Western blot analysis and calcium mobilization experiments on proximal tubular cells were performed as described previously (18). The $\mathrm{B} 2$ receptor $\mathrm{Ab}$ (mouse $\mathrm{mAb}$ B40820) used in the Western blot experiment (dilution 1/500) was from Transduction Laboratories (Lexington, Kentucky, USA).

Kidney protein extraction for enzyme activity measurements and zymography. For PA activity determination, one-sixth of a kidney is rapidly transferred into a 1.5$\mathrm{ml}$ reaction tube containing $150 \mu \mathrm{l}$ of ice-cold buffer A (13 mM sodium citrate, $0.33 \mathrm{M}$ sodium acetate, $\mathrm{pH}$ 3.9) and manually homogenized on ice for 10 seconds using a potter's device (1.5 $\mathrm{ml}$ Piston Pellet; Treff AG, Degersheim, Switzerland), followed by centrifugation for 5 minutes at $12,000 \mathrm{~g}$ at $4^{\circ} \mathrm{C}$. Supernatant $(100 \mu \mathrm{l})$ is then added to $5 \mu \mathrm{l}$ of $20 \%$ acetic acid, gently mixed, and stored at $-80^{\circ} \mathrm{C}$ until further use. For MMP-2 and MMP-9 zymography, one-sixth of a kidney is transferred into $150 \mu \mathrm{l}$ of ice-cold buffer (10 mM Tris$\mathrm{HCl}, \mathrm{pH}$ 7.5, $150 \mathrm{mM} \mathrm{NaCl}, 1 \mathrm{mM}$ EDTA, $1 \mathrm{mM}$ EGTA) in a $1.5-\mathrm{ml}$ reaction tube and treated as described for PA activity, but without addition of $5 \mu \mathrm{l}$ of $20 \%$ acetic acid. Protein concentrations were determined by the Bio-Rad Protein Assay (Bio-Rad Laboratories, Ivry sur Seine, France).

$P A$ activity and zymography. PA activity present in cell supernatants and kidney extracts was determined using a chromogenic assay (spectrolyse/fibrin; Biopool International, Umea, Sweden). This kit contains a specific EPA stimulator, Desafib-x. Samples were diluted in $0.9 \%$ of $\mathrm{NaCl}$ before analysis. MMP-2 and -9 activities were determined using zymography. Cell supernatant $(20 \mu \mathrm{l})$ or kidney extract $(25 \mu \mathrm{g})$ with nonreducing SDS sample buffer was loaded onto 8-10\% SDS-polyacrylamide gels containing $1 \mathrm{mg} / \mathrm{ml}$ gelatin. After electrophoresis, gels were soaked two times for 20 minutes in $2.5 \%$ (vol/vol) of Triton X-100 at room temperature and incubated overnight at $37^{\circ} \mathrm{C}$ in activation buffer $(10 \mathrm{mM}$ Tris- $\mathrm{HCl}, \mathrm{pH} 7.5$, $1.25 \%$ Triton $\mathrm{X}-100$ [vol/vol], $5 \mathrm{mM} \mathrm{CaCl}_{2}$, and $1 \mu \mathrm{M}$ $\mathrm{ZnCl}_{2}$ ). The following day, gels were stained for 3 hours with $0.25 \%$ (wt/vol) Coomassie brilliant blue in a mixture of methanol/acetic acid/water (50/10/40 $\mathrm{vol} / \mathrm{vol} / \mathrm{vol}$ ) and destained in the same mixture without Coomassie brilliant blue.

Statistical analysis. All the data are expressed as mean plus or minus SD. Statistical analyses were performed using SPSS software (SPSS Science, Chicago, Illinois, USA). ANOVA with post hoc Tukey alpha test was performed for comparison between the different groups. $P$ values less than 0.05 were considered statistically significant.

\section{Results}

UUO-induced tubulointerstitial fibrosis is significantly bigher in $\mathrm{B2}^{-/-}$mice. Interstitial collagen deposition was studied histomorphometrically in Sirius red-stained renal sections of $B 2^{-/-}$and $B 2^{+/+}$mice as an index of the fibrotic response to UUO.

Interstitial fibrosis increased progressively from day 1 to day 14 and became significantly different between the two mouse strains 3 days after obstruction (Figure 1a). At day 5, the difference between $B 2^{-/-}$and $B 2^{+/+}$ mice was the largest (Figure $1 \mathrm{~b}$ ), with an interstitial collagen expression that occupied $35 \% \pm 4 \%$ and $17 \% \pm 2 \%$ of the kidney sections, respectively. Since this difference was smaller at day 14 , and extreme tissue damage was observed at day 14 , all further experiments were carried out 5 days after obstruction. No significant difference between both mice strains in tubulointerstitial fibrosis of sham-operated and the contralateral kidney was observed (data not shown). PAS and hematoxylin-stained kidney sections showed an increase in tubular damage characterized by tubular dilation, atrophy, and hypercellularity in the obstructed kidneys, but without differences between $B 2^{-/-}$and $B 2^{+/+}$mice (not shown). 

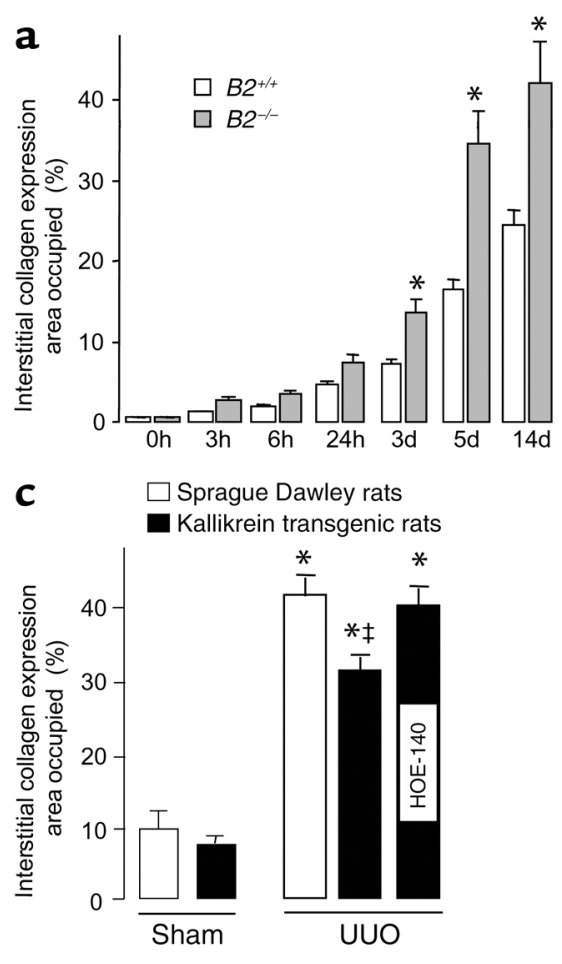

Further evidence of $B 2$ receptor-mediated reduction of tubulointerstitial fibrosis. To verify that the effect observed in $B 2^{-/-}$mice was effectively due to the absence of B2 receptor activation, we have performed UUO either on transgenic rats exhibiting increased endogenous bradykinin expression caused by overexpression of the human tissue kallikrein gene TGR(hKLK1) (15) or on $B 2^{+/+}$mice treated with a specific kinin-B2 receptor antagonist (HOE-140).

TGR(hKLK1) rats displayed significantly less UUOinduced tubulointerstitial fibrosis than their nontransgenic littermates (Figure 1c), which was abolished by HOE-140 treatment. Furthermore, HOE-140 also increased UUO-induced interstitial fibrosis in $\mathrm{B2}^{+/+}$ mice (Figure 1d). B2 $2^{-/-}$mice, the transgenic rat model, and pharmacological treatment thus strongly suggest that in both mice and rats, bradykinin $\mathrm{B} 2$ receptor activation reduces renal fibrosis.

Identification of ECM compounds. We next determined, using immunostaining, expression of ECM compounds known to be increased after UUO (Figure 2). After 5 days of UUO, the expression of fibronectin and collagen I, III, and IV was increased in both mouse strains. However, only collagen type III and IV expression was significantly higher in the $B 2^{-/-}$mice (Figure 2 , b and c, respectively).

How does bradykinin modify tubulointerstitial fibrosis? Analysis of $m R N A$ expression and enzyme activity. Current evidence supports the idea that fibrosis of several organs, including the kidney, is a consequence of the disturbance of the balance between ECM synthesis and degradation.

Using Northern blotting, we first studied mRNA expression of key proteins in UUO: ECM proteins

\section{Figure 1}

Quantitative analysis of UUO-induced renal interstitial collagen expression in $B 2^{-/-}$knockout mice, kallikrein transgenic rats, and $B 2$ receptor antagonist-treated wild-type mice. (a) Collagen expression in obstructed kidneys from $\mathrm{B2}^{+/+}$and $\mathrm{B} 2^{-/-}$mice after different periods of ureteral obstruction (3 hours to 14 days). Data are expressed as the mean \pm SD percentage of the interstitial area stained; $n=10 ;{ }^{*} P<0.05$ compared with respective $B 2^{+/+}$(same time of obstruction). (b) Representative photographs of Sirius red-stained renal sections of 5 day-obstructed and sham-operated $\mathrm{B2}^{+/+}$and $B 2^{-/-}$mice. Original magnification $\times 400$. (c) Collagen expression in kidneys from 5 day-obstructed and sham-operated kallikrein transgenic and control (Sprague Dawley) rats. One group was treated with HOE-140. $n=5$ per group. ${ }^{*} P<0.05 \mathrm{com}$ pared with sham-operated rats; $¥ P<0.05$ compared with UUO kallikrein transgenic rats without HOE-140. (d) Collagen expression in kidneys from 5 day-obstructed $\mathrm{B}^{+/+}$mice treated with saline only or with saline containing HOE-140 at $500 \mu \mathrm{g} / \mathrm{kg} / \mathrm{d}$; $n=10$ per group. Data expressed as the mean \pm SD percentage of the interstitial area stained; ${ }^{*} P<0.05$ compared to without HOE-140. (fibronectin and collagen I, III, and IV), TGF- $\beta$, monocyte chemoattractant peptide-1 (MCP-1), and osteopontin. UUO increased expression of all mRNA tested without difference between $B 2^{+/+}$and $B 2^{-/-}$mice, except for collagen IV mRNA expression, which was higher in $B 2^{-/-}$mice (Figure 3).

The two main systems involved in ECM degradation are the matrix metalloproteinases and the plasminogen activation system, which are closely interlinked (19). We thus investigated these degrading systems and found that UUO clearly increased mRNA expression of matrix metalloproteinases MMP-2 and MMP-9 and their respective inhibitors TIMP-1 and TIMP-2 (Figure 4), as well as the mRNA expression of $\mathrm{PPA}, \mathrm{uPA}$, and the PA inhibitor type 1 (PAI-1) (Figure 4a). However, no difference in mRNA expression was found between $B 2^{+/+}$ and $B 2^{-/-}$mice.

Since it has been reported that bradykinin is a potent stimulus for tPA secretion (20), we have measured total PA activity in sham-operated and obstructed kidneys of both mice strains. In preliminary experiments we have observed that the specific stimulator of tPA) increased PA activity by only three times (not shown), while it is described to increase tPA activity by 300 times. In a control reaction in which we added purified tPA to kidney extracts, Desafib-X increased by $240 \pm 15$ times PA activity, showing that the lack of increase in PA activity in kidney extracts is probably caused by a low tPA concentration. Non-Desafib$\mathrm{X}$-stimulated PA activity is thus a mixture of tPA and $\mathrm{uPa}$ activity. All reported activities in this study are non-Desafib-X-stimulated total PA activities. UUO induced an increase in PA activity in kidneys of both 
$B 2^{-/-}$and $B 2^{+/+}$mice. However, there was a significantly lower PA activity in the obstructed kidneys of $B 2^{-/-}$ mice (Figure $4 \mathrm{~b}$ ), whereas no difference between the two mouse strains in PA activity was detected in the sham-operated kidneys.

This decrease in PA activity in obstructed kidneys from $B 2^{-/-}$mice was paralleled with a lower MMP-2 activity in kidneys of $B 2^{-/-}$mice as assessed by zymography (Figure 4c); no difference between $\mathrm{B}^{+/++}$and $\mathrm{B2}^{-/-}$ mice was observed for MMP-9 (data not shown).

Since the previous in vivo experiments were based on the difference between the presence or absence of $\mathrm{B} 2$ receptors, we have used primary cultures of renal proximal tubular cells obtained from kidneys of $\mathrm{B2}^{+/+}$mice to verify if bradykinin via $\mathrm{B} 2$ receptor activation was able to stimulate PA activity (Figure 5a). Interestingly, we were not able to detect bradykinin-induced PA activity in tubular cells cultivated in standard culture medium, whereas lovastatin or TGF- $\beta$ (positive controls) were able to stimulate PA activity. However, pretreatment of the tubular cells with proinflammatory factors (TNF- $\alpha$ and IFN- $\gamma$ ) resulted in a bradykinin-induced PA activity, which was blocked by the specific $\mathrm{B} 2$ antagonist (HOE-140). The increased bradykinininduced PA activity after pretreatment of cells by the proinflammatory factors was not due to increased bradykinin B2 receptor expression (Western blot, Figure $5 \mathrm{~b}$ ) or coupling (calcium mobilization, Figure $5 c$ ). Pretreatment with a nitric oxide inhibitor did not block the bradykinin-induced PA activity (Figure $5 \mathrm{a}$ ). Bradykinin treatment of proximal renal tubular cells obtained from $B 2^{-/-}$mice did not display increased PA activity under the conditions described above (not shown).

Taken together, these data strongly suggest that bradykinin is reducing UUO-induced tubulointerstitial fibrosis by increasing the activity of enzymes involved in ECM degradation and by decreasing collagen IV mRNA expression.

Macrophage infiltration is decreased in $\mathrm{B}^{-/-}$mice. One of the early events in the development of tubulointerstitial fibrosis is tubulointerstitial inflammation and, more precisely, interstitial macrophage infiltration. In the sham-operated kidney and in the contralateral unobstructed kidney (not shown), a low level of macrophages was present in the tubulointerstitium, but no significant difference was found between both mouse strains. As expected, UUO induced a significant increase in interstitial macrophage infiltration, and, surprisingly, this macrophage infiltrate was found to be significantly lower in the obstructed kidney of $B 2^{-/-}$mice compared with $B 2^{+/+}$mice (Figure 6a).

Cell proliferation is not modified in $B 2^{-/-}$mice. Cell proliferation also being a hallmark of obstructive nephropathy, we evaluated renal cell proliferation by immunostaining for PCNA. A very small number of PCNA-positive cells were found in the sham-operat- ed kidney and in the contralateral unobstructed kidney (not shown), but UUO clearly increased both tubular and interstitial PCNA staining in the obstructed kidneys of both mice groups, however, without difference between $\mathrm{B2}^{-/-}$and $\mathrm{B2}^{+/+}$mice (Figure $6 \mathrm{~b}$ ). Using an $\mathrm{Ab}$ that recognizes $\alpha$-SMC, and thus myofibroblasts, on PCNA adjacent sections (Figure 6c), it was found that the majority of proliferating cells identified with PCNA were myofibroblasts, with no difference between the two mouse strains.
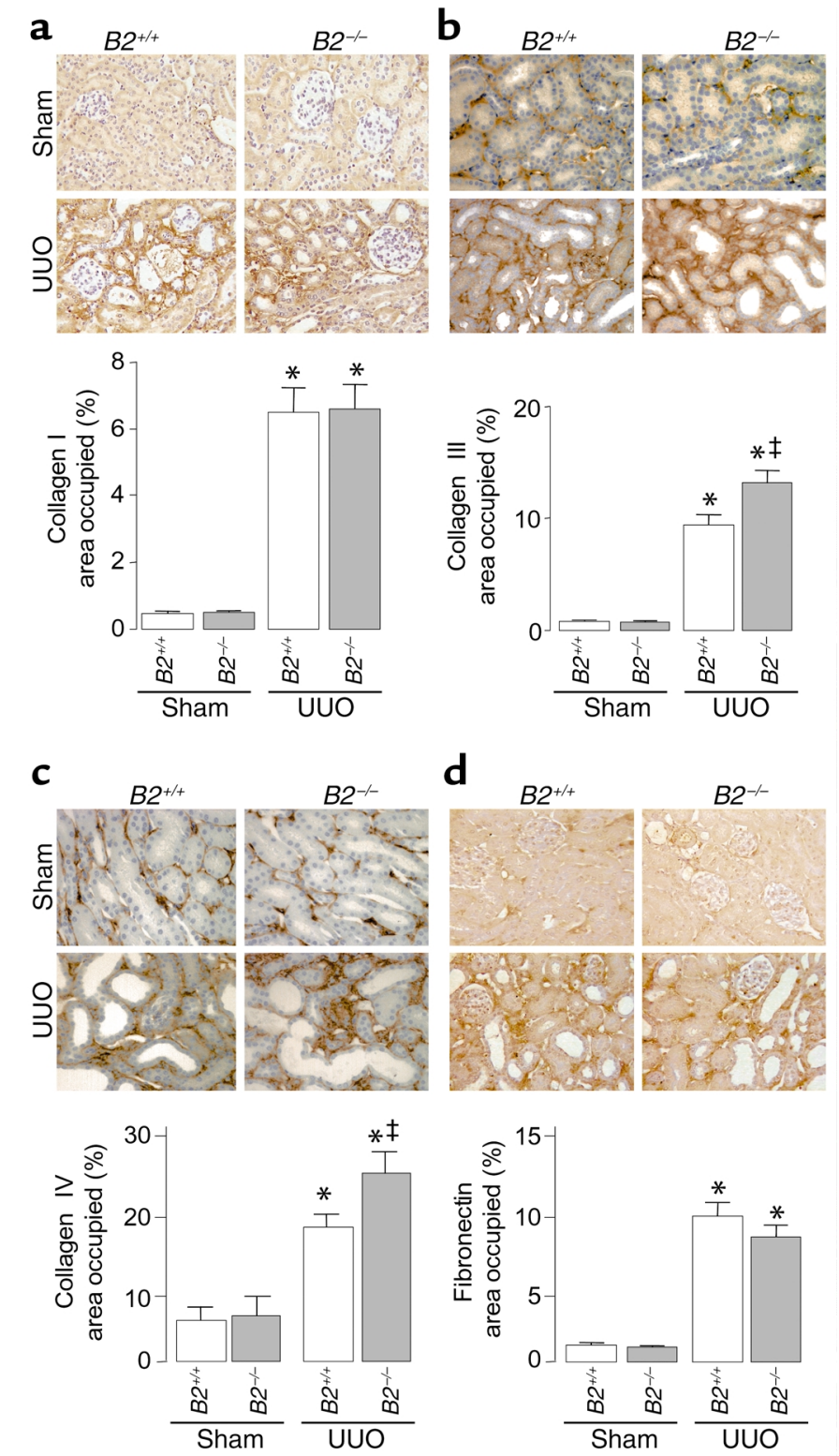

\section{Figure 2}

(a-d) Upper panels: representative light micrographs of immunodetection of collagen I, III, IV, and fibronectin expression within kidney sections from $\mathrm{B}^{+/+}$and $B 2^{-/-}$mice either sham-operated or UUO for 5 days. Lower panels, corresponding quantitative analysis of collagen I, III, IV, and fibronectin expression. Data are expressed as the mean \pm SD percentage of the interstitial area stained; $n=10 ;{ }^{*} P<0.05$ compared with respective sham-operated mice. $¥ P<0.05$ compared with obstructed $\mathrm{B}^{+/+}$mice. Original magnification $\times 400$. 
a

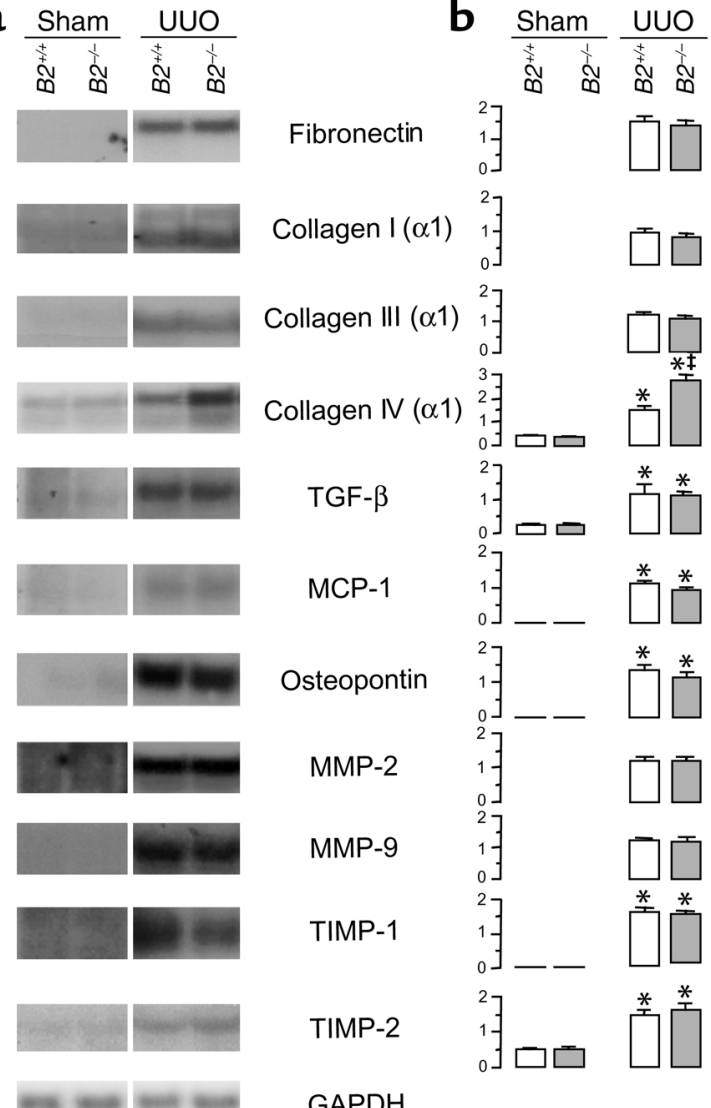

severe in $\mathrm{B2}^{+/+}$mice treated with a $\mathrm{B} 2$ receptor antagonist. In contrast, transgenic rats overexpressing kallikrein (thus expressing increased bradykinin levels) displayed less UUO-induced tubulointerstitial fibrosis, an effect that was blocked by $\mathrm{B} 2$ receptor antagonist administration. These observations are consistent with the antifibrotic effect of kallikrein overexpression on cardiac fibrosis (15) and with the observation that bradykinin levels are reduced in the obstructed rat kidney (25). By using immunohistochemistry, it was observed that the differences in tubulointerstitial fibrosis observed between $B 2^{-/-}$and $B 2^{+/+}$mice were mainly caused by differences in collagen III and IV expression. Collagen IV was the ECM protein that made up the largest part of the kidney sections compared with the other collagens. This is consistent with previous studies reporting a preferential accumulation of collagen IV in this obstructive nephropathy model (7).

ECM accumulation in pathological states is believed to result from an imbalance between both synthesis
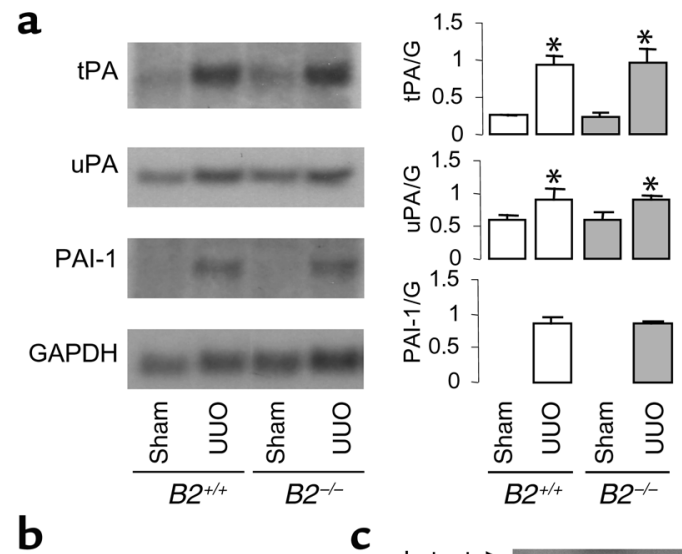

\section{Figure 3}

Northern-blot analysis of mRNA expression of total RNA of proteins involved in ECM formation and degradation and the matrix itself obtained from 5 day-obstructed and sham-operated kidneys of $\mathrm{B2}^{+/+}$ and $B 2^{-/-}$mice. (a) Representative autoradiograms. (b) Adjacent histograms are the mean densitometric values $( \pm S D)$ after analysis of the Northern blots and normalization with GAPDH; $n=10 .{ }^{*} P<0.05$ compared with respective sham-operated mice. $¥ P<0.05$ compared with $\mathrm{B2}^{+/+} \mathrm{UUO}$ mice.

\section{Discussion}

It is known that bradykinin is involved in the control of a wide range of physiological functions, including cardiovascular homeostasis (regulation of systemic blood pressure and organ blood flow), water and electrolyte transport, and pain-transmitting mechanisms (21). Besides these well-known physiological effects, bradykinin is involved in inflammation, and increasing clinical evidence suggests that bradykinin could play a role in the beneficial effect of ACE inhibitors in diabetic nephropathy and cardiovascular pathologies (22-24). In the present study we describe, we believe for the first time and in vivo, by using both genetically engineered animals and pharmacological tools, a bradykinin B2 receptor-dependent renal antifibrotic effect.

The bradykinin $B 2$ receptor and ECM accumulation. We have shown that B2-receptor activation in vivo reduced UUO-induced tubulointerstitial fibrosis. Renal fibrosis was more severe in $B 2^{-/-}$than in $B 2^{+/+}$mice and more

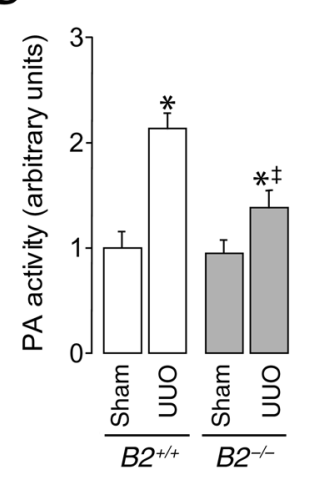

$\underset{\text { Latent }}{\rightarrow}$

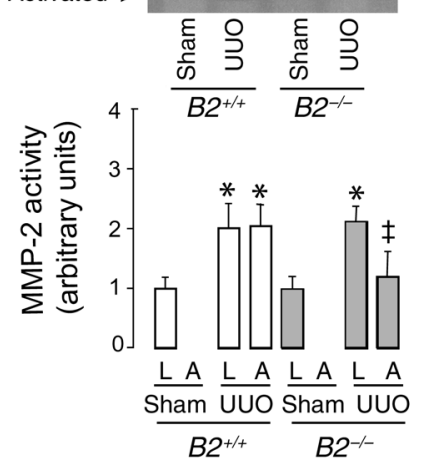

Figure 4

Expression and activity of the renal plasminogen system in 5 day-obstructed and sham-operated $B 2^{+/+}$and $B 2^{-/-}$mice. (a) Northern blot $m R N A$ expression analysis. Representative autoradiograms of tPA, uPA, PAI-1, and GAPDH are shown. Adjacent histograms are the mean densitometric values $( \pm S D)$ after analysis of the Northern blots $(n=10)$ and normalization with GAPDH. (b) PA activity. (c) Analysis of matrix MMP-2 activity by zymography. Quantification of MMP-2 activity by densitometric analysis is shown in the histogram. ${ }^{*} P<0.05$ compared with respective sham-operated mice. $¥ P<0.05$ compared with $B 2^{+/+}$UUO mice. $L$, latent; $A$, active MMP-2. 


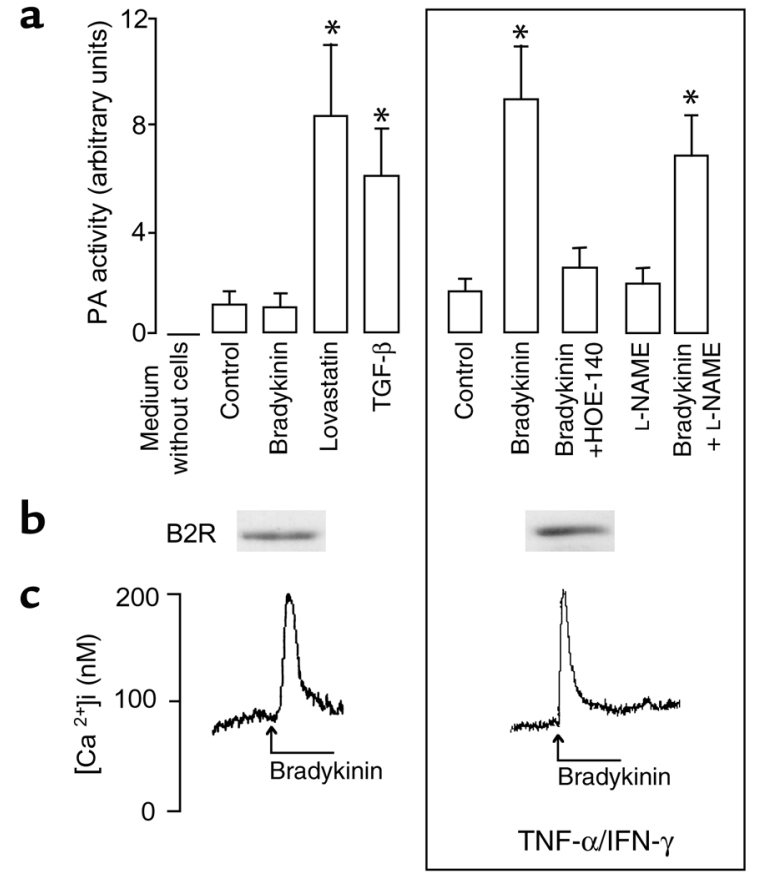

Figure 5

(a) Effect of bradykinin (24 hours at $0.1 \mu \mathrm{M}$ ) on PA activity from primary culture proximal tubular cells pretreated ( 24 hours) with TNF- $\alpha(150 \mathrm{U} / \mathrm{ml})$ and IFN- $\gamma(500 \mathrm{U} / \mathrm{ml})$ (boxed part of the figure) or non-pretreated. TGF- $\beta, 10 \mathrm{ng} / \mathrm{ml}$; HOE-140, $0.1 \mu \mathrm{M} ; N^{\mathrm{G}}$-nitroL-arginine methyl ester (L-NAME), $0.5 \mathrm{mM}$; lovastatine, $5 \mu \mathrm{M}$. (b) Western blot of B2 receptor (B2R) expression. (c) Increase in intracellular calcium $\left(\left[\mathrm{Ca}^{2+}\right]_{i}\right)$ following bradykinin $(0.1 \mu \mathrm{M})$ stimulation (time of stimulation 5 minutes). Values are mean \pm SEM of five different experiments. ${ }^{*} P<0.05$ compared with control cells. Representative blots or traces are shown.

and degradation (19). We analyzed how bradykinin B2 receptor activation can reduce ECM expression by studying both ECM synthesis and degradation.

First, mRNA expression of 14 proteins that have been shown to be involved/increased in this model (2) was studied. We observed in the obstructed kidney a net increase in mRNA expression of fibronectin, collagen I, III, and IV, TGF- $\beta$, MCP- 1 , osteopontin, MMP- 2 and -9 , TIMP-1 and -2 , as well as the plasminogen activation system (PAI-1, tPA, and uPA). However, no significant difference between $B 2^{-/-}$and $B 2^{+/+}$mice was observed except for collagen IV mRNA expression that was significantly higher in obstructed kidneys of the $B 2^{-/-}$ mice. The latter is consistent with the observed increased collagen IV protein expression. In vitro, bradykinin treatment either increased or decreased ECM production, depending on the cell type used. Bradykinin increased extracellular collagen synthesis by rat vascular smooth muscle cells (increased collagen I mRNA expression and gene-reporter activity, increased TIMP-1 protein expression, and increased TGF- $\beta$ protein expression) (26). On the other hand, in cultured cardiac fibroblasts, bradykinin decreased ECM production (collagen I, III, and fibronectin mRNA expression) by a mechanism probably involving nitric oxide production (27). Besides our in vivo study, no data are available on the effect of bradykinin on collagen IV (mRNA or protein) expression.

Second, we observed that $\mathrm{B} 2$ receptor activation increased PA activity. PA activity was significantly reduced in obstructed kidneys of $B 2^{-/-}$mice, and in primary culture, proximal tubular cells obtained from $\mathrm{B}^{+/+}$mice pretreated with inflammatory mediators, bradykinin via $\mathrm{B} 2$ receptor activation increased $\mathrm{PA}$ activity. In our system, PA activity is composed of both tPA and uPA activity. Although, to our knowledge, nothing is known about the effect of bradykinin on uPA activity, bradykinin was found to be one of the most potent stimuli of tPA release in the circulation in both rodents (20) and human vasculature (28). In this context it is important to stress that we determined PA activity and not tPA or uPA release as described above. How bradykinin modifies this PA activity and release is not known. In our study the decreased PA activity in $B 2^{-/-}$mice was not due to increased PAI-1 mRNA expression, since we observed the same increase in both mouse strains, which is con-

a

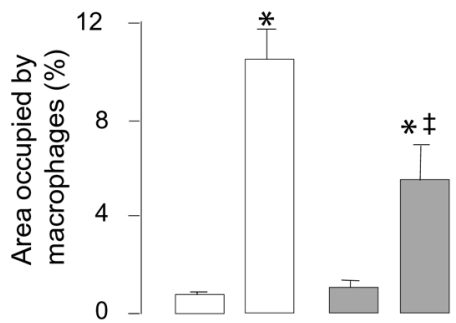

b

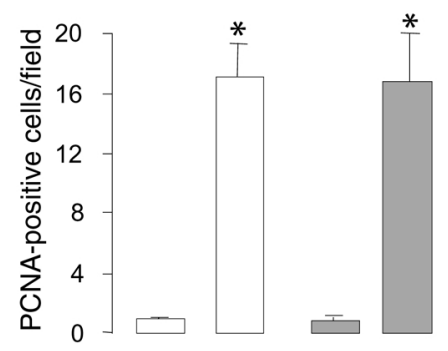

C

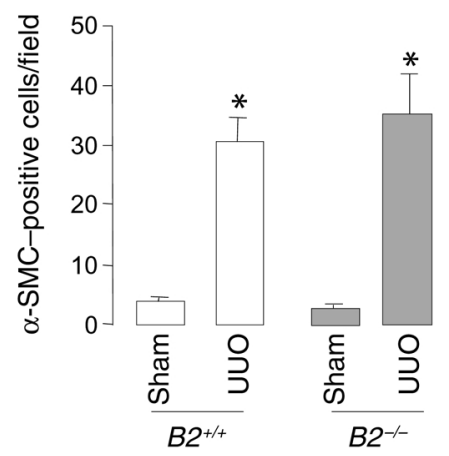

Figure 6

Immunohistochemical analysis of interstitial macrophage- (a), PCNA- (b), and $\alpha$-SMC-positive (c) cell expression in kidney sections from obstructed ( 5 day) and sham-operated kidneys of $B 2^{+/+}$and $B 2^{-/-}$mice. Data are expressed as the mean $\pm \mathrm{SD} ; n=10 ;{ }^{*} P<0.05$ compared with respective sham-operated mice. $¥ P<0.05$ compared with $\mathrm{B2}^{+/+}$UUO mice. 
sistent with an earlier report that bradykinin induces tPA but not PAI-1 release in human vasculature (28). In human vasculature bradykinin-induced tPA release was nitric oxide and cyclooxygenase independent (29). We found in primary culture proximal tubular cells from $\mathrm{B2}^{+/+}$mice that bradykinin-induced PA activity was nitric oxide independent. Another possibility for PA activation/secretion by bradykinin might be a direct interaction between the $\mathrm{B} 2$ receptor and the PAs. This was shown recently for the NMDA ( $N$-methyl-D-aspartate) receptor, which was found to directly interact with tPA. NMDA treatment induced tPA release from the NMDA receptor, which resulted in tPA-dependent cleavage of the NR1 subunit of the NMDA receptor, thereby increasing the receptor's activity (30). Such a direct interaction with the B2 receptor might explain the rapid tPA release upon bradykinin treatment in humans (29).

Third, decreased PA activity in $B 2^{-/-}$mice coincided with decreased MMP-2 activity. Collagen IV is the preferential substrate for MMP-2 (31). Plasmin can transform metalloproteinases from their latent to their active forms. Furthermore, uPA seems to be able to activate MMP-2 by activation of a membrane-bound metalloproteinase (19). The paralleled decrease in PA and MMP-2 activity related to increased ECM expression thus suggests a role for the $\mathrm{B} 2$ receptor/PA activity/MMP-2 activity cascade in ECM degradation.

Finally, the increased collagen IV mRNA expression can also be the result of the decreased PA activity in $B 2^{-/-}$mice since this will result in increased fibrin deposition that stimulates collagen accumulation (19).

Bradykinin and macrophage infiltration. Ureteral obstruction is primarily a nonimmune stimulus, but results in an interstitial immune infiltrate by the production of tubular-derived chemotactic factors as early as 4 hours after the onset of obstruction $(32,33)$. This infiltrate (mainly macrophages and lymphocytes) releases cytokines and chemokines, which can induce collagen synthesis by fibroblasts and cause further injury of tubules (2). Surprisingly, kidneys of UUO $B 2^{-/-}$mice exhibited a significantly lower number of interstitial macrophages than $B 2^{+/+}$mice. This reduced number of macrophages in the tubulointerstitium of obstructed kidneys of $B 2^{-/-}$compared with $B 2^{+/+}$mice, accompanied by increased tubulointerstitial ECM deposition, seems to contradict the role of this early event in the development of renal fibrosis after ureteral obstruction. It is, however, consistent with the welldescribed proinflammatory actions of bradykinin, mainly characterized by its vasodilatory effects and leukocyte accumulation $(34,35)$. In general, decreased interstitial macrophage infiltration is correlated to reduced tubulointerstitial fibrosis in the UUO model. There are, however, quite a few exceptions in the UUO model in which interstitial macrophage infiltration is not related to or has different effects on the degree of tubulointerstitial fibrosis. A reduction of around 50\% of infiltrating macrophages in the UUO model result- ed in a reduction of $10 \%$ and $25 \%$ of tubulointerstitial fibrosis in AT1 receptor-deficient mice (36) and treatment with a chemokine receptor antagonist (37), respectively, showing that the degree of macrophage infiltration was not correlated to the same degree of fibrosis. In another study it was shown that an AT2 receptor antagonist reduced interstitial fibrosis without an effect on interstitial macrophage infiltration (38). Furthermore, in our study reduced interstitial macrophage infiltration was not related to reduced activated fibroblast infiltration, which was observed in studies where reduced macrophage infiltration is accompanied by reduced interstitial fibrosis $(37,39)$. The absence of a positive correlation between decreased macrophage infiltration and decreased tubulointerstitial fibrosis in our study might thus have at least two origins: (a) the absence of differences in activated fibroblast infiltration between $\mathrm{B2}^{+/+}$and $\mathrm{B2}^{-/-}$ mice, and (b) the reduction of about $35 \%$ of infiltrating macrophages in $\mathrm{B2}^{-/-}$mice, which does not compensate the reduced PA activity in this strain, thus resulting in increased tubulointerstitial fibrosis compared with $B 2^{+/+}$mice.

Taken together, the results of this study show that bradykinin B2 receptor activation in vivo reduces UUO-induced tubulointerstitial fibrosis most likely by increasing the activity of a PA/MMP-2 cascade. These observations favor a potential role of bradykinin in the antifibrotic effects of ACE inhibitors.

1. Klahr, S., Schreiner, G., and Ichikawa, I. 1988. Progression in renal disease. N. Engl. J. Med. 318:1657-1666.

2. Klahr, S. 1998. Obstructive nephropathy. Kidney Int. 54:286-300.

3. Nath, K.A. 1994. Tubulointerstitial changes as a major determinant in the progression of renal damage. Am. J. Kidney Dis. 264:F874-F881.

4. Chevalier, R.L., and Klahr, S. 1988. Therapeutic approaches in obstructive uropathy. Semin. Nephrol. 18:652-658.

5. Chevalier, R.L., Thornhill, B.A., and Chang, A.Y. 2000. Unilateral ureteral obstruction in neonatal rats leads to renal insufficiency in adulthood. Kidney Int. 58:1987-1995.

6. Ishidoya, S., Morrissey, J., McCracken, R., Reyes, A., and Klahr, S. 1995. Angiotensin II receptor antagonist ameliorates renal tubulointerstitial fibrosis caused by unilateral ureteral obstruction. Kidney Int. 47:1285-1294.

7. Kaneto, H., Morrissey, J., McCracken, R., Reyes, A., and Klahr, S. 1994. Enalapril reduces collagen IV synthesis and expansion of the interstitium in the obstructed rat kidney. Kidney Int. 45:1637-1647.

8. Erdös, E.G. 1990. Angiotensin I converting enzyme and the changes in our concepts through the years. Hypertension. 16:363-370.

9. Jaspard, E., Wei, L., and Alhenc-Gélas, F. 1993. Differences in the properties and enzymatic specificities of the two active sites of angiotensin I-converting enzyme (kininase II). J. Biol. Chem. 268:9496-9503.

10. Campbell, D.J., Kladis, A., and Duncan, A.M. 1994. Effects of converting enzyme inhibitors on angiotensin and bradykinin peptides. Hypertension. 23:439-449.

11. Heart Outcomes Prevention Evaluation Study (HOPE) investigators. 2000. Effects of ramipril on cardiovascular and microvascular outcomes in people with diabetes mellitus: results of the HOPE study and MICRO-HOPE substudy. Lancet. 355:253-259.

12. Gainer, J.V., Morrow, J.D., Loveland, A., King, D.J., and Brown, N.J. 1998. Effect of bradykinin-receptor blockade on the response to angiotensinconverting-enzyme inhibitor in normotensive and hypertensive subjects. N. Engl. J. Med. 339:1285-1292.

13. Pitt, B., et al. 2000. Effect of losartan compared with captopril on mortality in patients with symptomatic heart failure: randomised trial - the losartan heart failure survival study ELITE II. Lancet. 355:1582-1587.

14. Borkowski, J.A., et al. 1995. Targeted disruption of a B2 bradykinin receptor gene in mice eliminates bradykinin action in smooth muscle and neurons. J. Biol. Chem. 270:13706-13710.

15. Silva, J.A., et al. 2000. Reduced cardiac hypertrophy and altered blood 
pressure control in transgenic rats with the human tissue kallikrein gene. FASEB. J. 14:1858-1860.

16. Marin-Castaño, M.E., et al. 2002. Induction of functional bradykinin B1-receptors in normotensive rats and mice under chronic angiotensin converting enzyme inhibitor treatment. Circulation. 105:627-632.

17. Bergin, E., Levine, J.S., Koh, J.S., and Lieberthal, W. 2000. Mouse proximal tubular cell-cell adhesion inhibits apoptosis by a cadherin-dependent mechanism. Am. J. Physiol. 278:F758-F768.

18. Schanstra, J.P., et al. 1998. The B1-agonist [des-Arg10]-kallidin activates transcription factor NF- $\mathrm{\kappa B}$ and induces homologous upregulation of the bradykinin B1-receptor in cultured human lung fibroblasts. J. Clin. Invest. 101:2080-2091.

19. Rerolle, J.P., Hertig, A., Nguyen, G., Sraer, J.D., and Rondeau, E.P., 2000. Plasminogen activator inhibitor type 1 is a potential target in renal fibrogenesis. Kidney Int. 58:1841-1850.

20. Smith, D., Gilbert, M., and Owen, W.G. 1985. Tissue plasminogen activator release in vivo in response to vasoactive agents. Blood. 66:835-839.

21. Margolius, H.S. 1995. Kallikreins and kinins. Hypertension. 26:221-229.

22. Lewis, E.J., Hunsicker, L.G., Bain, R.P., and Rohde, R.D. 1993. The effect of angiotensin-converting-enzyme inhibition on diabetic nephropathy. N. Engl. J. Med. 329:1456-1462.

23. Geiger, H. 1997. Are angiotensin II receptor blockers superior to angiotensin converting enzyme inhibitors with regard to their renoprotective effect? Nephrol. Dial. Transplant. 12:640-642.

24. Remme, W.J. 1997. Bradykinin-mediated cardiovascular protective actions of ACE-inhibitors. Drugs. 54(Suppl.):59-70.

25. El-Dahr, S.S., et al. 1993. Upregulation of renin-angiotensin system and downregulation of kallikrein in obstructive nephropathy. Am. J. Physiol. 264:F874-F878.

26. Douillet, C.D., et al. 2000. Mechanisms by which bradykinin promotes fibrosis in vascular sooth muscle cells: role of TGF- $\beta$ and MAPK. Am.J. Physiol. 279:H2829-H2837.

27. Kim, N.N., Villegas, S., Summerour, R.S., and Villareal, F.J. 1999. Regulation of cardiac fibroblast extracellular matrix production by bradykinin and nitric oxide. J. Mol. Cell. Cardiol. 31:457-466.

28. Brown, N.J., Gainer, J.V., Stein, C.M., and Vaughan, D.E. 1999. Bradykinin stimulates tissue plasminogen activator release in human vasculature. Hypertension. 33:1431-1435.

29. Brown, N.J, Gainer, J.V., Murphey, L.J., and Vaughan, D.E. 2000. Bradykinin stimulates tissue plasminogen activator release from human forearm vasculature through $\mathrm{B}(2)$ receptor-dependent, NO synthase-independent, and cyclooxygenase-independent pathways. Circulation. 102:2190-2196.

30. Nicole, O., et al. 2001. The proteolytic activity of tissue-plasminogen activator enhances NMDA receptor-mediated signaling. Nature Med. 7:59-64.

31. Morgunova, E., et al. 1999. Structure of human pro-matrix metalloproteinase-2: activation mechanism revealed. Science. 284:1667-1670.

32. Ong, A.C.M., and Fine, L.G. 1994. Loss of glomerular function and tubulointerstitial fibrosis: cause or effect? Kidney Int. 45:345-351.

33. Schreiner, G.F., Harris, K.P., Purkerson, M., and Klahr, S. 1988. Immunological aspects of acute ureteral obstruction: immune cell infiltrate in the kidney. Kidney Int. 34:487-493.

34. Burch, R., Farmer, S.G., and Steranka, L.R. 1990. Bradykinin receptor antagonists. Med. Res. Rev. 10:237-269.

35. Koyama, S., et al. 2000. Bradykinin stimulates lung fibroblasts to release neutrophil and monocyte chemotactic activity. Am. J. Respir. Cell. Mol. Biol. 22:75-84.

36. Satoh, M., et al. 2001. Renal interstitial fibrosis is reduced in angiotensin II type 1a receptor deficient mice. J. Am. Soc. Nephrol. 12:317-325.

37. Anders, H.J., et al. 2001. A chemokine receptor CCR-1 antagonist reduces renal fibrosis after unilateral ligation. J. Clin. Invest. 109:251-259. doi:10.1172/JCI200214040.

38. Morrissey, J.J., and Klahr, S. 1999. Effect of AT2 receptor blockade on the pathogenesis of renal fibrosis. Am. J. Physiol. 276:F39-F45.

39. Oda, T., et al. 2001. PAI-1 deficiency attenuates the fibrogenic response to ureteral obstruction. Kidney Int. 30:587-596. 